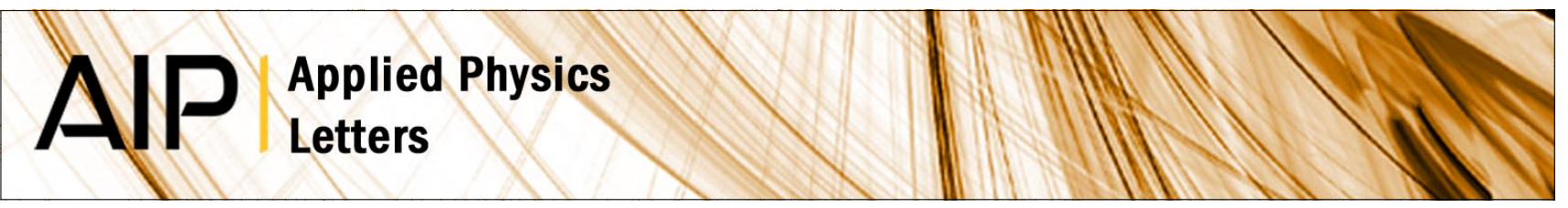

\title{
Phase-locked loop based on nanoelectromechanical resonant-body field effect transistor
}

\author{
S. T. Bartsch, A. Rusu, and A. M. Ionescu
}

Citation: Appl. Phys. Lett. 101, 153116 (2012); doi: 10.1063/1.4758991

View online: http://dx.doi.org/10.1063/1.4758991

View Table of Contents: http://apl.aip.org/resource/1/APPLAB/v101/i15

Published by the American Institute of Physics.

\section{Related Articles}

Origin of kink effect in $\mathrm{AIGaN} / \mathrm{GaN}$ high electron mobility transistors: Yellow luminescence and Fe doping Appl. Phys. Lett. 101, 153505 (2012)

Poole Frenkel current and Schottky emission in SiN gate dielectric in AIGaN/GaN metal insulator semiconductor heterostructure field effect transistors

Appl. Phys. Lett. 101, 153504 (2012)

Hybrid vertical transistor based on controlled lateral channel overflow

J. Appl. Phys. 112, 074509 (2012)

Field-effect diode based on electron-induced Mott transition in $\mathrm{NdNiO3}$

Appl. Phys. Lett. 101, 143111 (2012)

Solution-processed dye-sensitized $\mathrm{ZnO}$ phototransistors with extremely high photoresponsivity

J. Appl. Phys. 112, 074507 (2012)

\section{Additional information on Appl. Phys. Lett.}

Journal Homepage: http://apl.aip.org/

Journal Information: http://apl.aip.org/about/about_the_journal

Top downloads: http://apl.aip.org/features/most_downloaded

Information for Authors: http://apl.aip.org/authors

\section{ADVERTISEMENT}

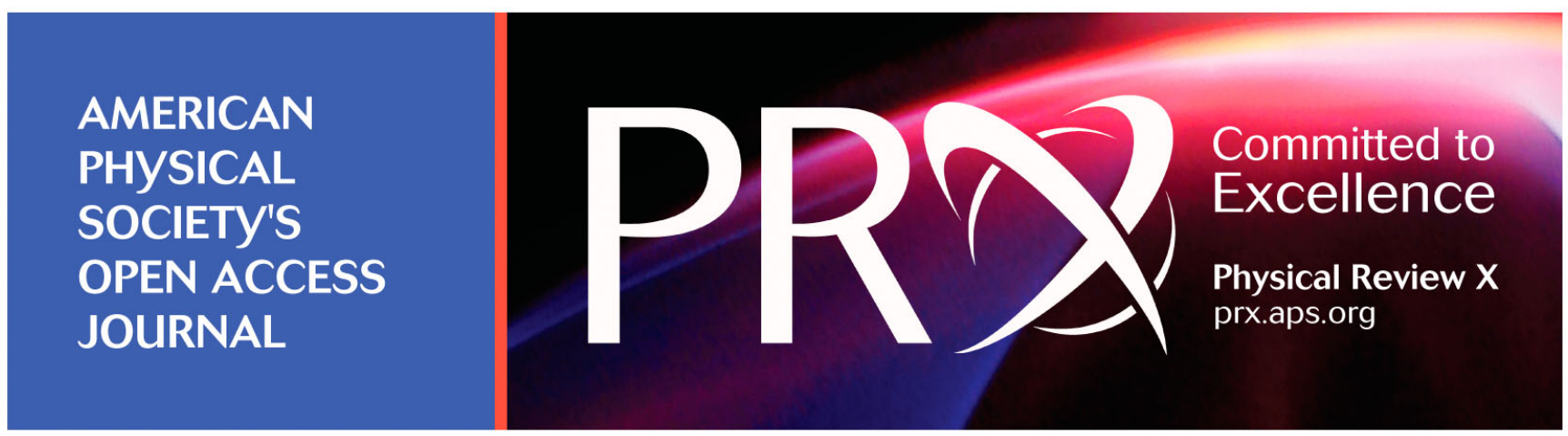




\title{
Phase-locked loop based on nanoelectromechanical resonant-body field effect transistor
}

\author{
S. T. Bartsch, A. Rusu, and A. M. Ionescu ${ }^{\text {a) }}$ \\ Nanoelectronic Devices Laboratory, Ecole Polytechnique Fédéral de Lausanne (EPFL), 1015 Lausanne, \\ Switzerland
}

(Received 17 August 2012; accepted 28 September 2012; published online 11 October 2012)

\begin{abstract}
We demonstrate the room-temperature operation of a silicon nanoelectromechanical resonant-body field effect transistor (RB-FET) embedded into phase-locked loop $(P L L)$. The very-high frequency resonator uses on-chip electrostatic actuation and transistor-based displacement detection. The heterodyne frequency down-conversion based on resistive FET mixing provides a loop feedback signal with high signal-to-noise ratio. We identify key parameters for PLL operation, and analyze the performance of the RB-FET at the system level. Used as resonant mass detector, the experimental frequency stability in the ppm-range translates into sub atto-gram $\left(10^{-18} \mathrm{~g}\right)$ sensitivity in high vacuum. The feedback and control system are generic and may be extended to other mechanical resonators with transistor properties, such as graphene membranes and carbon nanotubes. (C) 2012 American Institute of Physics. [http://dx.doi.org/10.1063/1.4758991]
\end{abstract}

Nanoelectromechanical systems (NEMS) are of great interest both for applications in technology and fundamental sciences. As basic building block for frequency clocks, ${ }^{1}$ mixer-filters, ${ }^{2}$ or super-abrupt switches, ${ }^{3}$ NEMS are promising for future low power, mobile communication, and signal processing systems. ${ }^{4}$ Nanomechanical resonators have emerged as a valuable tool to observe and prove quantum mechanics on macroscopic objects. ${ }^{5}$ With shrinking device dimensions and increasing frequency of operation, NEMS are also exploited as sensors with unprecedented sensitivities. The detection of mass at the single molecule level ${ }^{6}$ or force in the atto-Newton ( $\mathrm{aN})^{7}$ range has been demonstrated, which offers a great potential for applications in nanomechanical mass spectrometry ${ }^{8}$ and scanned probed sensing. ${ }^{9}$

In many sensor and timekeeping applications, it is essential to provide a feedback architecture which is capable of sustaining the mechanical resonance and/or tracking its frequency in real-time. In frequency clocks, for example, the electromechanical resonator, such as a quartz crystal, is embedded in an oscillator circuit, which continuously sustains the natural frequency of oscillation. In resonant mass sensing, an induced frequency shift is translated back into an equivalent mass accreted onto the sensor element. More generally, embedding mechanical resonators into a closed-loop feedback architecture helps to study their performance at the system level, such as the phase noise and frequency stability, and explore failure and aging mechanisms in NEMS.

In this letter, we describe the room-temperature operation of silicon nanoelectromechanical, resonant-body field effect transistors (RB-FETs) embedded into an analog phase-locked loop $(P L L)$. In contrast to other previous work, ${ }^{7,10-12}$ the device operation and feedback scheme have some unique properties. First, the NEMS-PLL is realized using a fully integrated transduction at very high frequencies $(>100 \mathrm{MHz})$ by means of on-chip electrostatic actuation and transistor-based motion detection of clamped-clamped beam

\footnotetext{
${ }^{\text {a) }}$ Author to whom correspondence should be addressed. Electronic mail: adrian.ionescu@epfl.ch. Telephone: +41 216933978.
}

resonators. Second, the NEM resonator is used here as a resistive FET mixer, ${ }^{13}$ and thereby assumes the functional role of an active component at the system level. Further, the device is monolithically integrated in a fully depleted (FD-) silicon-on-insulator (SOI-) CMOS technology platform. This offers unique opportunities for hybridization of resonator arrays with CMOS circuitry on a single chip.

A SEM graph of a representative device is shown in Figure 1(a). The resonator is designed as doubly clamped beam, which is actuated via the lateral gate electrode (G1) and an electrostatic nano-gap. The second electrode is not used in this experiment $(\mathrm{G} 2=0 \mathrm{~V})$. The drain $(\mathrm{D})$, source $(\mathrm{S})$, and channel region form the n-type enhancement mode RB-FET. The FET characteristics and principle of operation of this device have been reported in previous work. ${ }^{13}$ The RB-FET here represents an advanced device generation with aggressively scaled dimensions. The resonator fabrication was based on an SOI-wafer with a $40 \mathrm{~nm}$ thin silicon device layer and structured via hybrid e-beam/DUV lithography, followed by a typical HF-vapor release process. The details on the fabrication and possible co-integration with CMOS are reported elsewhere. ${ }^{14}$ Table I summarizes the most relevant parameters of the device used in this work.

The operation of the NEMS-PLL is based on a twosource, heterodyne frequency down-conversion combined with a lock-in detection (Stanford Research SR-830). This method has been introduced to preserve a high peak-to-background, including the phase information, of the resonance signal in RB-FETs. Figure 1(b) shows the open loop response of the resonator and the fundamental, in-plane flexural resonance at $\Omega_{0} / 2 \pi=103 \mathrm{MHz}$. The extracted quality factor is $Q=470$. The device was probed in a Suss MicroTech vacuum chamber. Figure 2 shows the NEMS-PLL system diagram. The RB-FET is depicted as 3-terminal device. The two voltage-controlled oscillators (RF, LO) generate a signal at $\Omega$ and $\Omega+\Omega_{\text {ref }}$, respectively, which are split into two branches ( $\Omega$ is the circular frequency). The signals are attenuated and routed to the drain (D) and gate $(\mathrm{G})$ terminal. Operated as resistive mixer, the RB-FET generates output 

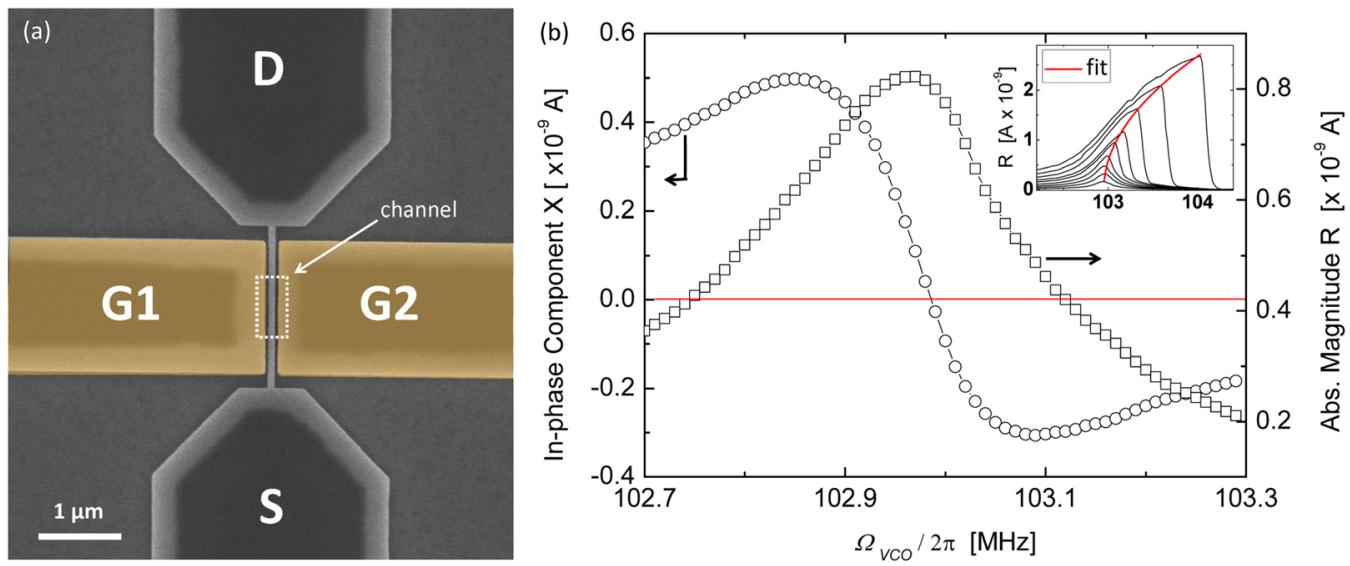

FIG. 1. (a) SEM graph of the clamped-clamped beam nanomechanical resonant-body FET. The lateral gate eletrodes (G1, G2) are indicated in false color. The drain (D), the channel, and the source (S) form the $\mathrm{n}^{+} \mathrm{pn}^{+}$-type enhancement mode FET. The under-etch of the buried oxide after release is visible. (b) The resonance is detected in open loop configuration. The absolute magnitude $R=\sqrt{X^{2}+Y^{2}}$ and the in-phase component $\mathrm{X}$ are plotted versus the swept frequency. The red line is the zero-reference level for the X-component, which is nulled-out at maximum peak response by tuning the lock-in phase to $\Phi=-83^{\circ}$. The drive power was set to $P_{R F}=-31 \mathrm{dBm}$ and $P_{L O}=-66 \mathrm{dBm}$. Inset: absolute magnitude R versus the swept frequency with increasing driving strength $\left(P_{R F}\right.$ from -40 to $-16 \mathrm{dBm}$ ), showing the typical response of a Duffing oscillator. The red-line is the "backbone" fit. The data are used to determine the onset to nonlinearity $\left(\mathrm{P}_{R F, c r}\right)$ and the system dynamic range $(1.6 \mathrm{~Hz}$ measurement bandwidth).

signals at the intermediate frequency $\Omega_{r e f}$. The two quadratures $(\mathrm{X}, \mathrm{Y})$ are generated by the two phase-sensitive detectors (PSD) of the lock-in unit. These components are essentially dependent on the amplitude of the signal $V_{s i g}$ (i.e., on the FET properties, the transduction gap, and the mechanical displacement), and the phase offset $\Phi \Delta$ between the signal and the reference:

$$
\begin{aligned}
& X \propto V_{\text {sig }} \cos \left(\Omega_{r e f} t+\Delta \Phi\right) \\
& Y \propto V_{\text {sig }} \sin \left(\Omega_{r e f} t+\Delta \Phi\right),
\end{aligned}
$$

where $\Delta \Phi=\varphi_{\text {ref }}-\varphi_{\text {sig }}$.
In order to close the loop and track the resonance frequency, an adequate feedback signal is required. From the resonator's response function (forced, damped harmonic oscillator), it is evident that a $\pi$-phase shift of the input drive is introduced relative to the resonator's output signal. This phase shift is well reflected by the down-converted, low-frequency component at $\Omega_{r e f}$. By use of a tunable phase shifter, the lock-in quadratures can be nulled-out at resonance, and thereby used to generate a feedback signal that is linear (with a negative slope) in the vicinity of the resonance frequency (see Figure 1(b)). The control signal is routed back to the VCXO input control ports, hence closing the loop. ${ }^{15}$

\begin{tabular}{|c|c|c|}
\hline Parameter & Symbol & Value \\
\hline Resonance frequency & $\Omega_{0} / 2 \pi[\mathrm{MHz}]$ & 103 \\
\hline Dimensions $^{\mathrm{a}}$ & $W[\mathrm{~nm}] \times T[\mathrm{~nm}] \times L[\mu \mathrm{m}]$ & $88 \times 45 \times 1.89$ \\
\hline Oxide thickness & $T_{o x}[\mathrm{~nm}]$ & 16 \\
\hline Transduction gap & $d_{g a p}[\mathrm{~nm}]$ & $\sim 60$ \\
\hline $\mathrm{D} / \mathrm{S} / \mathrm{G}$ doping & $n^{+}\left[\mathrm{cm}^{-3}\right]$ & $>10^{19}$ \\
\hline Channel doping & $p\left[\mathrm{~cm}^{-3}\right]$ & $\sim 1 \times 10^{16}$ \\
\hline Estimated channel length & $L_{c h}[\mu \mathrm{m}]$ & $\sim 0.4$ \\
\hline Quality factor & $Q$ & 470 \\
\hline Modal mass ${ }^{\mathrm{a}}$ & $m_{e f f}[\mathrm{fg}]$ & 13.9 \\
\hline LO power & $P_{L O}[\mathrm{dBm}]$ & -66 \\
\hline RF Power (onset mech. nonlinearity) & $P_{R F, c r}[\mathrm{dBm}]$ & -24 \\
\hline Operating pressure & $P[\mathrm{mbar}]$ & $1 \times 10^{-5}$ \\
\hline Operating temperature & $T[\mathrm{~K}]$ & 300 \\
\hline Reference frequency & $\Omega_{r e f} / 2 \pi[\mathrm{kHz}]$ & $<100$ \\
\hline Gate voltage & $V_{G}[\mathrm{~V}]$ & 10 \\
\hline Operating dyn. range ${ }^{\mathrm{b}}$ & $D R[\mathrm{~dB}]$ & $\sim 43$ \\
\hline Mass responsivity & $\mathcal{R}[\mathrm{Hz} / \mathrm{zg}]$ & $\sim 23$ \\
\hline Fract. frequency stability ${ }^{c}$ & $\langle\delta \Omega / \Omega\rangle_{\tau=1 \mathrm{~s}}[\mathrm{ppm}]$ & $\sim 8.92$ \\
\hline Mass noise floor ${ }^{\mathrm{c}}$ & $\delta m[\mathrm{zg}]$ & $\sim 250$ \\
\hline Pract. mass resolution ${ }^{\mathrm{c}}$ & $\delta \mathcal{M}[\mathrm{ag}]$ & $<2.5$ \\
\hline
\end{tabular}

TABLE I. Relevant experimental parameters of the RB-FET are listed.

${ }^{a}$ The dimensions can be accurately determined via SEM and TEM imaging.

${ }^{\mathrm{b}}$ The dynamic range is defined as ratio of the $r m s$ amplitude reproducing predominantly linear response to the $r m s$ noise floor at resonance, and was determined with the help of the inset of Figure 1(b).

${ }^{\mathrm{c}}$ Value referred to $1.6 \mathrm{~Hz}$ measurement bandwidth and a total loop gain of $\mathrm{G}=3.76$. 


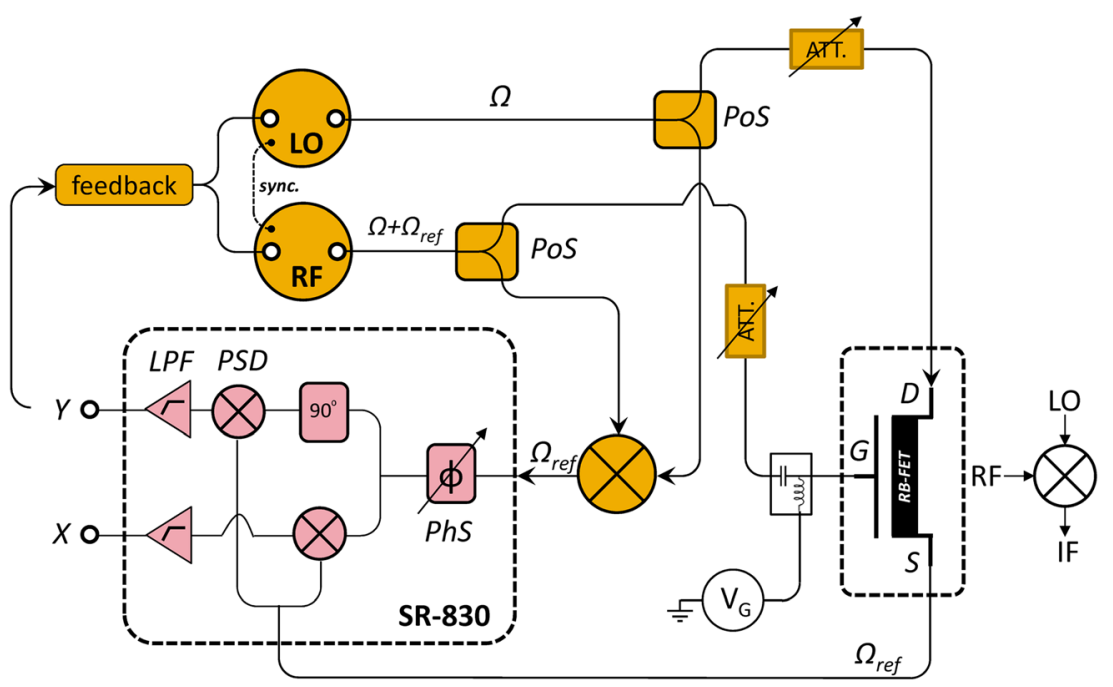

FIG. 2. Experimental system diagram and schematic of the measurement circuit. Shown is the closed-loop case; for open loop resonance detection, the feedback branch is interrupted. The signal sources (RF, LO) are synchronized, divided via power splitters (PoS), attenuated (ATT) and routed to the resonator and the reference mixer, respectively. The lock-in amplifier is indicated with the most relevant components, including phase shifter $(\mathrm{PhS})$, phase-sensitive detector (PSD), amplification stages, and low-pass filter (LPF). The RB-FET is used as 3-terminal, resistive FET mixer (RF/LO/IF).

The tracking edge is reached at the point where the slope of the feedback characteristics falls to zero. Hence, the resonator's natural line width will determine the tracking bandwidth. We note that other mixing techniques based on FM or AM demodulation schemes ${ }^{16}$ produce quadratures that are symmetric around resonance and are less suited to realize analog NEMS-PLLs.

We now turn our analysis to the steady-state response of the NEMS-PLL. ${ }^{17}$ The VCXO generates an output whose frequency is a function of the control voltage $V_{\text {cont }}$ :

$$
\Omega_{P L L}=\Omega_{V C O}+\mathrm{G}_{V C O} \mathrm{~V}_{c o n t},
$$

where $\Omega_{V C O}$ is the free-running frequency (zero feedback) and $G_{V C O}$ the VCXO gain $(\mathrm{kHz} / \mathrm{V})$. The control voltage $V_{\text {cont }}$ depends on feedback gain $G_{P S D}(\mathrm{mV} / \mathrm{kHz})$ and the phase offset $\Delta \Phi$ :

$$
\mathrm{V}_{\text {cont }}=\mathrm{G}_{P S D} \Delta \Phi
$$

The VCXO frequency is set equal to the resonator's center frequency, $\Omega_{V C O}=\Omega_{0}$, and the phase $\Delta \Phi$ is manually tuned so that no error signal is generated. The closed loop is then described by

$$
\Omega_{P L L}=\Omega_{V C O}+\mathrm{G}_{V C O} \mathrm{G}_{P S D}\left(\Omega_{0}-\Omega_{P L L}\right) .
$$

If the resonance frequency changes by $\Delta \Omega=\Omega^{\prime}{ }_{0}-\Omega_{0}$, then the PLL frequency follows:

$$
\Omega_{P L L}=\Omega_{0}+\frac{G}{1+G} \Delta \Omega,
$$

where $G=G_{V C O} G_{P S D}$ is the total loop gain. The VCXO gain is set to $50 \mathrm{kHz} / \mathrm{V}$. The feedback gain depends on the properties of the RB-FET (e.g., signal strength $V_{\text {sig }}$, quality factor Q), and the sensitivity value $S$ manually set at the lock-in unit. The gain $G_{P S D}$ can be measured and determined by linearly approximating the feedback signal around resonance, as shown in Figure 3(a). The total loop gain $G$ can be determined experimentally by shifting the VCXO center frequency $\Omega_{V C O}$ and reading out the loop frequency $\Omega_{P L L}$, as shown in Figure 3(b). From the linear slope, the value of $G$ can be obtained. The measured and the calculated values for the total loop gain are compared in Table II, with excellent agreement.

We monitor the loop frequency in real-time using a directional coupler and a frequency counter (Agilent 53131A, sampling time $1 \mathrm{~s}$ ). This allows to study the frequency stability of the RB-FET-NEMS at the system level. From Eq. (5), it is clear that the frequency fluctuations are reflected in $\Omega_{P L L}$ and directly scale with the total loop gain. Figure 3(c) shows the frequency fluctuation versus the loop gain, and the according functional fit. Equation (5) also tells us that the higher the loop gain (i.e., $G /(1+G) \rightarrow 1$ ), the more reliably the $P L L$ tracks the shift in the resonance frequency. The loop response time depends on the time constant of the loop filter, which was typically set to $100 \mathrm{~ms}$ (or $1.6 \mathrm{~Hz}$ ) in this letter.

We tested the tracking behavior by programming a voltage-controlled frequency shift. In a capacitive actuation scheme, the electrical spring constant is negative, and the electrostatic nonlinearity leads to the reduction of the resonant frequency. Figure 4(a) shows the PLL frequency vs. time when changing the gate voltage in steps of $\Delta \mathrm{V}_{\mathrm{G}}=20 \mathrm{mV}$. The resulting frequency shifts agree reasonably well with the frequency tunability obtained from open loop measurements $\left(\partial \Omega_{0} / 2 \pi \partial V_{G} \sim 300 \mathrm{kHz} / \mathrm{V}\right)$. We note that a small error remains to this method, as a change in $\mathrm{V}_{\mathrm{G}}$ will also change the signal strength, which is a parameter of the feedback signal (see Eqs. (1) and (3)).

For many NEMS applications, the frequency stability is key. In the time domain, the Allan variance ${ }^{18}$ is a widely accepted standard for the frequency stability. Figure 4(b) shows the instantaneous rms fractional frequency fluctuations of the RB-FET resonator as function of the integration time $\tau \geq 1 \mathrm{~s}$. The Allan variance can be improved by further reducing the measurement bandwidth, until random walk sets in $\left(\tau \geq 10 \mathrm{~s}, \sigma_{A}(\tau) \propto \tau^{0.5}\right)$. The investigation of $\sigma_{\mathrm{A}}(\tau)$ for shorter integration times is limited by the data acquisition time of the GPIB connection ( $\sim 100 \mathrm{~ms}$ ) (ideally, zero deadtime between measurements is required).

For resonant mass sensing, the mass resolution is limited by the minimum resolvable frequency shift of the whole measurement system. By inspection of the resonator 

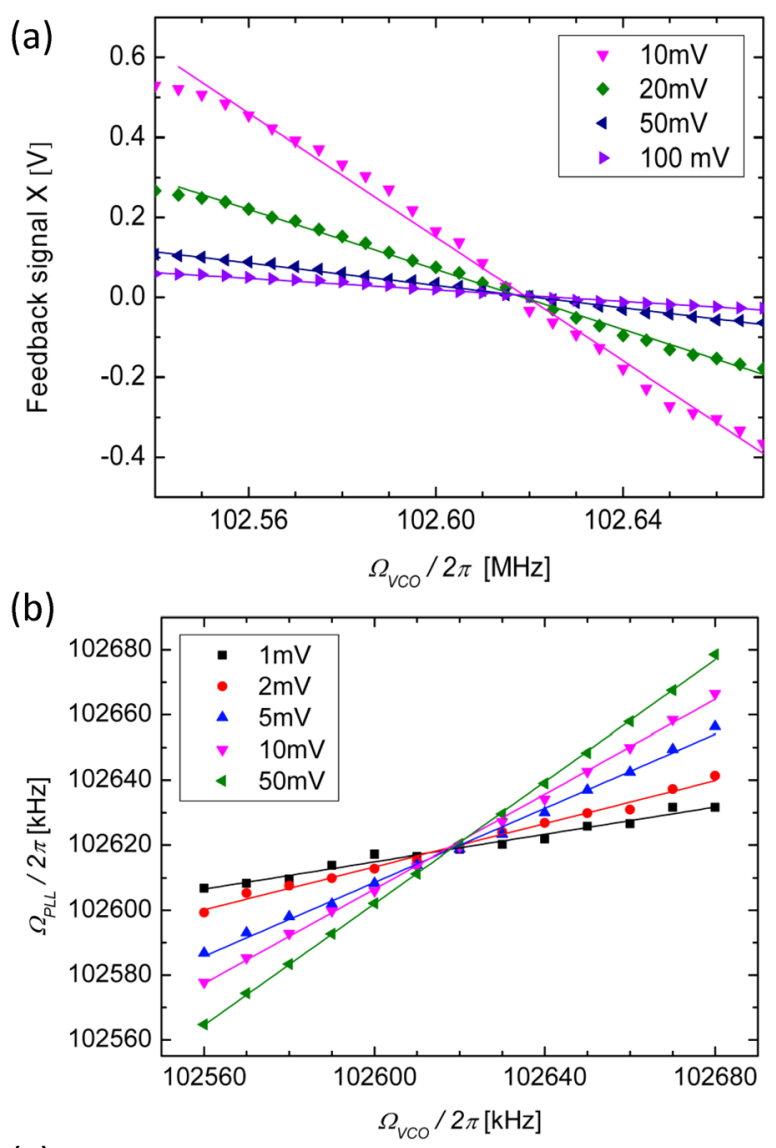

(c)

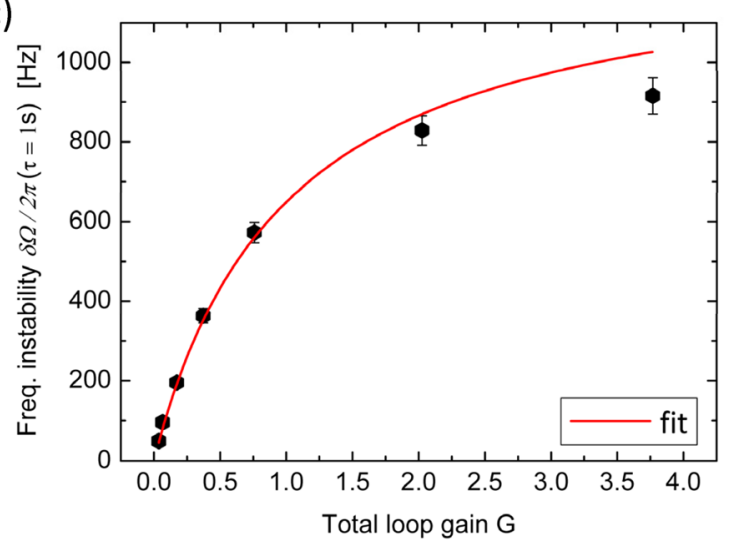

FIG. 3. (a) The feedback signal $X$ plotted versus the frequency in the vicinity of the mechanical resonance. The lines are a fit. The sensitivity is set as parameter, which determines the slope (the gain $\mathrm{G}_{\mathrm{PSD}}$ ) of the feedback signal. (b) The PLL-frequency is plotted versus the VCO frequency. The sensitivity is set as parameter. The lines are a fit. From its slope, the total gain $\mathrm{G}$ is deduced. (c) The frequency instability for $1 \mathrm{~s}$ integration time is plotted versus the total loop gain G. For the fit, the functional dependence $k \cdot \frac{G}{1+G}$ of Eq. (4) is used ( $k$ is a fitting constant).

dimensions (SEM and TEM imaging), the resonator mass can be determined to $m_{0} \approx 13.9 \mathrm{fg}$. For the fundamental mode response, the modal mass is $m_{\text {eff }}=0.735 m_{0}$, which yields an estimated mass responsitivity of $\mathcal{R}=-\omega_{0} / 2 m_{\text {eff }} \approx 23 \mathrm{~Hz} / \mathrm{zg}$ for this device. The measured frequency noise floor translates into a mass detection noise floor $\langle\delta m\rangle=\mathcal{R}^{-1}\langle\delta \Omega\rangle$, which is about $\sim 250 \mathrm{zg}$ (for $\tau=1 \mathrm{~s}$ and $\mathrm{G}=3.76$ ). This device should enable a practical mass sensor in the attogram range at room temperature in high vacuum (see also Table I).

The frequency fluctuations result from the sum of all stochastic noise sources intrinsic (thermo mechanical fluctu-
TABLE II. Comparison of measured and calculated gain values relevant for the PLL operation.

\begin{tabular}{lcccccccc}
\hline \hline Parameter & \multicolumn{7}{c}{ Value } \\
\hline VCO gain $\mathrm{G}_{\mathrm{VCO}}$ & & \multicolumn{7}{c}{50} \\
$\mathrm{kHz} / \mathrm{V}]$ & 1 & 2 & 5 & 10 & 20 & 50 & 100 \\
Sensitivity S [mV] & 75 & 40.4 & 15.3 & 7.73 & 3.84 & 1.403 & 0.719 \\
Feedback gain G $\mathrm{PSD}_{\mathrm{PS}}$ & & & & & & & \\
{$[\mathrm{mV} / \mathrm{kHz}]$} & & & & & & \\
Total loop gain G/calc. & 3.75 & 2.0235 & 0.765 & 0.387 & 0.192 & 0.0702 & 0.0359 \\
Total loop gain G/meas. & 3.76 & 2.0232 & 0.758 & 0.373 & 0.173 & 0.0667 & 0.0369 \\
\hline \hline
\end{tabular}

ation) and extrinsic (instrumentation, readout circuitry) to the resonator. It can be shown that the frequency noise density is inversely proportional to the carrier signal to noise ratio. ${ }^{19,20}$ This allows an estimation of the mass resolution based on separately measured, experimental parameters (see Table I)

$$
\langle\delta m\rangle=\frac{m_{e f f}}{2 Q} \sqrt{1 / \tau} \times 10^{(-D R / 20)}
$$

which yields $\langle\delta m\rangle \approx 100 \mathrm{zg}$ (for a dynamic range of $43 \mathrm{~dB}$ ), and is close to the value obtained from the measured frequency fluctuations. It can be expected that the lower limit is not set by the resonator intrinsic noise, but by the posttransducer noise (read-out) of the specific NEMS-PLL setup.

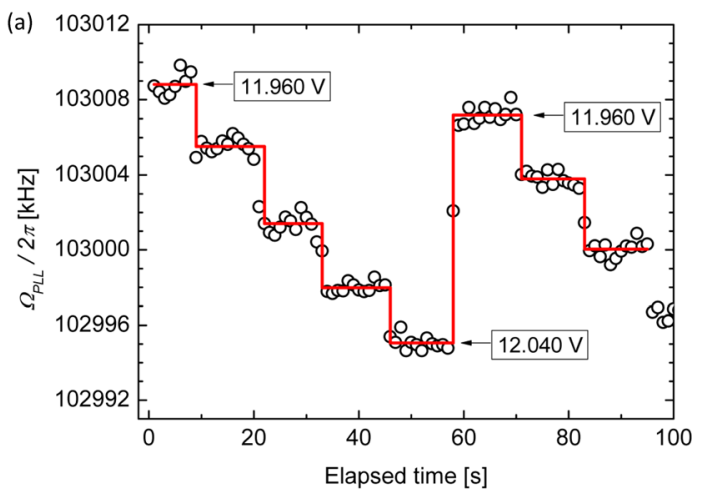

(b)

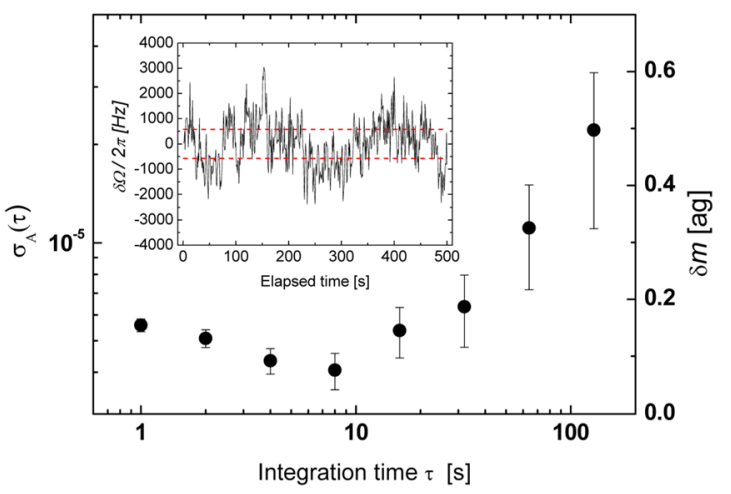

FIG. 4. (a) Real-time frequency tracking of a silicon nanoelectromechanical RB-FET. The resonance frequency is shifted by repeatedly ramping the gate voltage from $11.960 \mathrm{~V}$ to $12.040 \mathrm{~V}$ is steps of $20 \mathrm{mV}$. The loop frequency is monitored with a frequency counter. The loop gain was set to 3.76 . The red line corresponds to the mean value over time for each voltage value and is a guide to the eye. The induced frequency shift corresponds to the voltage tunability of $\sim 300 \mathrm{kHz} / \mathrm{V}$ of the NEMS resonator. (b) The Allan variance for a feedback gain of $G=2.02$. The right axis shows the corresponding mass noise floor, with a practical mass resolution in the atto-gram range. For longer time constants, the frequency drift deteriorates the stability. Inset: frequency stability referred to the nominal drive frequency over time. 
We suggest and discuss therefore some aspects for optimization on the system level. Revisiting Figure 2, it would be more efficient to run the PLL with only one VCXO. The small, but non-zero discrepancy between gains of the VCXOs result in an additional source of noise, which is reflected in the IF component. We measured a $25 \%$ reduction of the Allan variance when providing feedback to one VCXO (RF) only. A one-source PLL may be realized by use of a single sideband modulator, which generates together with the lock-in reference signal the component at $\Omega+\Omega_{\text {ref }}$ (and not $\Omega \pm \Omega_{r e f}$ in case of a regular mixer). However, single sideband modulators require input $90^{\circ}$ quadrature hybrids, which are typically available only at frequencies $>1$ $\mathrm{MHz}$. The use of lock-in detectors operating at $\mathrm{VHF}^{21}$ or signal processing in the digital domain could bypass this problem. Further, we observed that a systematic and consistent grounding of the DUT and the instrumentation can help to further improve frequency stability. ${ }^{22}$

In conclusion, we have demonstrated the VHF operation of a monolithic, silicon nanoelectromechanical RB-FET embedded into a phase-locked loop. The device represents the most scaled generation of its kind, combined with an allelectronic, fully integrated transduction scheme. The $P L L$ implementation allows the study of the frequency stability of resonant FETs at the system level. Used as mass detector, we project a mass resolution in the atto-gram range in high vacuum at room temperature. Better performance can be expected upon optimization of the system, or with parametric mechanical amplification. ${ }^{23}$ This low-noise frequency control scheme is generic and can be extended to any NEMS with FET properties, e.g., resonant carbon nanotubes, graphene membranes, or compound semiconductor resonators. As such, it may emerge as valuable tool for studying basic noise properties ${ }^{24}$ in NEMS devices, and enable real-time mass and force sensing.

This work has been partially funded by the FP7 project NEMSIC. We thank C. Dupre and E. Ollier for device fabrication.
${ }^{1}$ B. P. Otis and J. M. Rabaey, IEEE J. Solid-State Circuits 38, 1271 (2003). ${ }^{2}$ S. T. Bartsch, A. Rusu, and A. M. Ionescu, Nanotechnology 23, 225501 (2012).

${ }^{3}$ N. Abele, R. Fritschi, K. Boucart, F. Casset, P. Ancey, and A. M. Ionescu, Tech. Dig. Int. Electron Devices Meet. 2005, 479.

${ }^{4}$ C. T. C. Nguyen, in Proceedings of the IEEE International Frequency Control Symposium (2005), p. 951.

${ }^{5}$ J. D. Teufel, T. Donner, D. L. Li, J. W. Harlow, M. S. Allman, K. Cicak, A. J. Sirois, J. D. Whittaker, K. W. Lehnert, and R. W. Simmonds, Nature 475, 359 (2011).

${ }^{6}$ J. Chaste, A. Eichler, J. Moser, G. Ceballos, R. Rurali, and A. Bachtold, Nat. Nanotechnol. 7, 300 (2012).

${ }^{7}$ M. Li, H. X. Tang, and M. L. Roukes, Nat. Nanotechnol. 2, 114 (2007).

${ }^{8}$ A. K. Naik, M. S. Hanay, W. K. Hiebert, X. L. Feng, and M. L. Roukes, Nat. Nanotechnol. 4, 445 (2009).

${ }^{9}$ D. Rugar, R. Budakian, H. J. Mamin, and B. W. Chui, Nature 430, 329 (2004).

${ }^{10}$ Y. T. Yang, C. Callegari, X. L. Feng, K. L. Ekinci, and M. L. Roukes, Nano Lett 6, 583 (2006).

${ }^{11}$ X. L. Feng, R. R. He, P. D. Yang, and M. L. Roukes, in Transducers '07 \& Eurosensors XXI, Digest of Technical Papers (2007), Vols. 1 and 2, p. U167.

${ }^{12}$ K. L. Ekinci, X. M. H. Huang, and M. L. Roukes, Appl. Phys. Lett. 84, 4469 (2004)

${ }^{13}$ S. T. Bartsch, A. Lovera, D. Grogg, and A. M. Ionescu, ACS Nano 6, 256 (2012).

${ }^{14}$ E. Ollier, C. Dupre, G. Arndt, J. Arcamone, C. Vizioz, L. Duraffourg, E. Sage, A. Koumela, S. Hentz, G. Cibrario et al., in IEEE International Conference on Micro Electromechanical Systems (2012), p. 1368.

${ }^{15}$ We note that it is desirable to keep $\Omega_{\text {ref }}$ constant during $P L L$ operation. Hence, the feedback signal is routed back to both VCXOs. The resonator is driven mainly by the RF-source $(-31 \mathrm{dBm})$, the LO-signal is heavily attenuated $(-66 \mathrm{dBm})$.

${ }^{16}$ V. Gouttenoire, T. Barois, S. Perisanu, J. L. Leclercq, S. T. Purcell, P. Vincent, and A. Ayari, Small 6, 1060 (2010).

${ }^{17}$ B. Razavi, Design of Monolithic Phase-Locked Loops and Clock Recovery Circuits-A Tutorial (IEEE, New York, 1996).

${ }^{18}$ D. W. Allan, Proc. Inst. Electr. Eng. 54, 221 (1966).

${ }^{19}$ K. L. Ekinci, Y. T. Yang, and M. L. Roukes, J. Appl. Phys. 95, 2682 (2004).

${ }^{20}$ W. P. Robins, Phase Noise in Signal Sources (Peter Peregrinus Ltd., 1982).

${ }^{21}$ See, e.g., Zurich Instruments or Standford Research Systems.

${ }^{22}$ Signal grounding is specific to the experimental setup and therefore not treated here in detail. We observed one order of magnitude in frequency stability by grounding the wafer chuck.

${ }^{23}$ A. N. Cleland, New J. Phys. 7, 235 (2005).

${ }^{24}$ K. Y. Fong, W. H. P. Pernice, and H. X. Tang, Phys. Rev. B 85, 161410 (2012). 